\title{
Eclipsing binary stars with extreme light curve asymmetries mined from large astronomical surveys
}

\author{
Athanasios Papageorgiou ${ }^{1,2, \star}$, Georgios Kleftogiannis ${ }^{3, \star \star}$ and Panagiota-Eleftheria \\ Christopoulou ${ }^{3, \star \star \star}$ \\ ${ }^{1}$ Pontificia Universidad Católica de Chile, Instituto de Astrofísica, Av. Vicuña Mackenna 4860, 782-0436 \\ Macul, Santiago, Chile \\ ${ }^{2}$ Millennium Institute of Astrophysics, Santiago, Chile \\ ${ }^{3}$ University of Patras, Department of Physics, 26500, Patra, Greece
}

\begin{abstract}
The O'Connell effect is one of the most perplexing challenges in binary studies as it has not been convincingly explained. Furthermore, a simple method to obtain essential parameters for eclipsing binaries exhibiting this effect and to extract information describing the asymmetry in the light curve maxima is needed. We have developed an automated program that characterizes the morphology of light curves by depth of both minima, height of both maxima and curvature outside the eclipses.
\end{abstract}

\section{Introduction}

In the last few decades, due to the automatic ground-based and space telescopes, the amount of data has increased dramatically. In order to search for peculiar light curves, automatic pipelines are needed to deal with this amount of data. Further to our search ([4]) we apply an improved version of our automated method in several databases in order to detect Light Curve Asymmetries (LCA). The program is being developed in Python (https://www.python.org/).

\section{Method - results}

The program reads from a given catalog (name, period $P, V_{\max }$, etc.) of a specific database and applies two different sets of constraints ([4]) which were improved as follows. Each data point is examined to be valid according to the local variance of its neighbors in a certain phase bin. Combining this method with $\sigma$-clipping, the outliers were better defined and the performance of fitting improved accordingly. Then the program constructs the phase-magnitude diagram in the range $-0.25-1.25$ where a second order polynomial is fitted (Fig. 1), using the NumPy package (http://www.numpy.org/). Finally, fitting alert flags were included in order to control the goodness of fitting.

The method was applied in 13987 eclipsing binaries (EB) light curves from ASAS, OGLE II, OGLE III, NSVS and OMC databases. Of those 345 were found with strong out of eclipse variations

\footnotetext{
$\star$ apapageo@astro.puc.cl

$\star \star$ gkleftis@upatras.gr

$\star \star \star$ pechris@physics.upatras.gr
} 

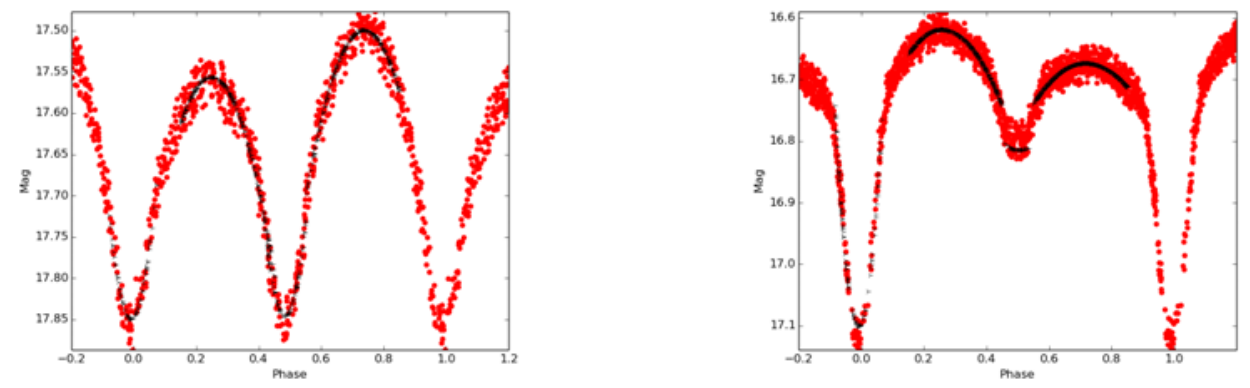

Figure 1. Contact EB OGLE-GD-ECL-07791 (left) and semi-detached EB OGLE-GD-ECL-03789 (right) with light curve asymmetries. Black points denote the polynomial fitting.

Table 1. A sample of 5 eclipsing binary stars from the new catalogue with strong LCA

\begin{tabular}{lclll}
\hline ID & $\mid$ MaxII - MaxI $\mid(\mathrm{mag})$ & $P(\mathrm{~d})$ & RA (h:m:s) & Dec (d:m:s) \\
\hline OGLE-GD-ECL-09205 & 0.0743 & 0.278594 & $13: 15: 52.74$ & $-64: 34: 52.1$ \\
OGLE-GD-ECL-10742 & 0.0751 & 0.291847 & $13: 31: 28.61$ & $-64: 08: 21.0$ \\
OGLE-GD-ECL-08412 & 0.0757 & 0.396209 & $11: 55: 07.87$ & $-61: 45: 31.8$ \\
OGLE-GD-ECL-07791 & 0.0567 & 0.363248 & $11: 33: 42.20$ & $-60: 36: 44.9$ \\
OGLE-GD-ECL-03789 & 0.0547 & 0.490414 & $10: 51: 12.11$ & $-60: 48: 38.7$ \\
\hline
\end{tabular}

$(\mid$ MaxI-MaxII $\mid>0.025 \mathrm{mag})$ as shown in Table 1. From ASAS, 120 EBs were classified as Eclipsing Contact type (EC) and 23 had multiple classification ([7, 8]); from OGLE II, 3 EBs were classified as Eclipsing W UMa type (EW) and 2 had multiple classification ([9, 10]); from OGLE III, 189 EBs were classified as EC ([2, 5, 6]); from NSVS, 4 EBs were classified as W UMa type ([3]); and from OMC, 4 EBs had multiple classification ([1]).

Acknowledgments: A.P. gratefully acknowledges the support provided by Fondecyt through grant \#3160782.

\section{References}

[1] Alfonso-Garzon, J., Domingo, A., Mas-Hesse, J.M., \& Gimenez, A., A\&A, 548, A79 (2012)

[2] Graczyk, D., Soszyński, I., Poleski, R., et al., Acta Astron., 61, 103 (2011)

[3] Hoffman D. I., Harrison T. E., \& McNamara B. J., AJ, 138, 2 (2009)

[4] Papageorgiou, A., Kleftogiannis, G., \& Christopoulou, PE., Contrib. Astron. Obs. Skalnate Pleso, 43, 3 (2014)

[5] Pawlak, M., Graczyk, D., Soszynski, I., et al., Acta Astron., 63, 323 (2013)

[6] Pietrukowicz, P., Mroz, P., Soszynski, I., et al., Acta Astron., 63, 115 (2013)

[7] Pojmanski, G., \& Maciejewski, G., Acta Astron., 54, 153 (2004)

[8] Pojmanski, G., Pilecki, B., \& Szczygiel, D., Acta Astron., 55, 275 (2005)

[9] Wyrzykowski, L., Udalski, A., Kubiak, M., et al., Acta Astron., 53, 1 (2003)

[10] Wyrzykowski, L., Udalski, A., Kubiak, M., et al., Acta Astron., 54, 1 (2004) 